\title{
ON PROJECTIVE PRIME IDEALS IN $C(X)$
}

\section{J. GLENN BROOKSHEAR}

\begin{abstract}
This note presents characterizations of the projective prime ideals in $C(X)$ and of the hereditary and semihereditary rings of continuous functions.
\end{abstract}

This note presents a characterization of the projective prime ideals in $C(X)$, the ring of real-valued continuous functions on a completely regular Hausdorff space $X$. This characterization is then applied to obtain a characterization of the hereditary rings of continuous functions. The reader is referred to [5] and [2] for background. The referee has pointed out that the results in this paper can also be derived from the more general results appearing in [4].

LEMMA. Each projective prime ideal in $C(X)$ is generated by an idempotent.

Proof. The following argument shows that a projective prime ideal is finitely generated. The lemma then follows from Theorem 3 of [3].

Suppose $P$ is a nonfinitely generated projective ideal in $C(X)$. By Theorem 2.4 of [2], $P$ is generated by a family $\left\{f_{\alpha}\right\}_{\alpha \in A}$ such that $\left\{\operatorname{coz} f_{\alpha}\right\}_{\alpha \in A}$ is star-finite. There is a countably infinite subset $\left\{Y_{i}\right\}_{i=1}^{\infty} \subseteq\left\{\operatorname{coz} f_{\alpha}\right\}_{\alpha \in A}$ such that $Y_{i} \cap Y_{j}=\varnothing$ if $i \neq j$. Now, by the complete regularity of $X$, for each $i$ there is a $g_{i} \in C(X)$ such that $0 \leqslant g_{i} \leqslant \frac{1}{2^{i}}$ and $\varnothing \neq \operatorname{coz} g_{i} \subseteq Y_{i}$. Neither $\sum_{i=1}^{\infty} g_{2 i}$ nor $\sum_{i=1}^{\infty} g_{2 i+1}$ can be a finite linear combination of elements of $\left\{f_{\alpha}\right\}_{\alpha \in A}$ since $\left\{\operatorname{coz} f_{\alpha}\right\}_{\alpha \in A}$ is star-finite. However, both sums are in $C(X)$ and their product is $0 \in P$. Thus, $P$ is not prime.

If $x \in X$, let $M_{x}$ denote the maximal ideal of $C(X)$ consisting of functions whose zero-sets contain $x$.

THEOREM 1. A proper ideal in $C(X)$ is a projective prime ideal if and only if it has the form $M_{x}$ for some isolated $x \in X$.

Proof. If $x \in X$ is isolated, then $M_{x}$ is a summand of $C(X)$ and hence projective.

Suppose $P$ is a proper projective prime ideal in $C(X)$. By the previous lemma, $P$ is principal and hence fixed since it is proper. Thus, it is contained in a fixed maximal ideal. But $P$ is contained in only one maximal ideal [5, 2.11 ], so the idempotent generating $P$ must be the characteristic function of

Received by the editors May 4, 1977 and, in revised form, September 14, 1977.

AMS (MOS) subject classifications (1970). Primary 54C40; Secondary 34B15.

(1) American Mathematical Society 1978 
$X \backslash\{x\}$ for some $x \in X$. Thus, $P=M_{x}$ for some isolated $x \in X$.

In [1] Bergman presents characterizations for the hereditary and semihereditary commutative rings which can be applied to produce the following results. However, the application of the results in [2] and the preceding theorem provide a more straightforward and revealing approach in the restricted setting of $C(X)$.

First, it was shown in [2] that a principal ideal in $C(X)$ is projective if and only if the support of the generating function is open. Moreover, if $X$ is basically disconnected, then every finitely generated ideal in $C(X)$ is principal [5, 14N.4 and 14.25]. Thus, $C(X)$ is semihereditary if and only if $X$ is basically disconnected.

THEOREM 2. The following are equivalent.

(a) $C(X)$ is hereditary.

(b) Every prime ideal is projective.

(c) Every maximal ideal is projective.

(d) $X$ is finite and discrete.

Proof. Clearly (a) implies (b) which implies (c). Moreover, if all maximal ideals are projective, they must be fixed at isolated points by Theorem 1. Thus, $X$ must be compact and discrete. Consequently, (c) implies (d). Finally, if $X$ is finite and discrete, every ideal in $C(X)$ is a summand so (d) implies (a).

\section{REFERENCES}

1. G. M. Bergman, Hereditary commutative rings and centres of hereditary rings, Proc. London Math. Soc. 23 (1971), 214-236.

2. J. G. Brookshear, Projective ideals in rings of continuous functions, Pacific J. Math. 71 (1977), 313-333.

3. G. De Marco, On the countably generated z-ideals of $C(X)$, Proc. Amer. Math. Soc. 31 (1972), 574-576.

4.

5. L. Gillman and M. Jerison, Rings of continuous functions, Van Nostrand, New York, 1960.

Department of Mathematics, Marquette University, Milwaukee, Wisconsin 53233 